\title{
Selectivity in Higher-Order Childbearing in Sweden
}

\author{
GUNNAR ANDERSSON, Ph.D, Associate Professor \\ Demography Unit, Department of Sociology, University of Stockholm, \\ Sweden
}

\begin{abstract}
In this note, we present relative risks of giving birth for mothers with different numbers of children. We use Swedish register data and study the propensity to continue the childbearing beyond child number two. We pay special attention to births of those higher orders that are studied only rarely in conventional demographic analyses. As the parity increases to higher numbers, we expect to find some kind of selection so that the group of mothers increasingly consists of very birth prone women. For births after a fourth child, we indeed find such an effect in that the relative risk of giving birth to an additional child then increases with the birth order. In our intensity-regression models, we also check whether this selection effect of increasing birth risks can be picked up by the inclusion of a specific factor for unobserved heterogeneity in the mothers' propensity to give birth. We find that the positive gradient in the propensity to give birth indeed disappears when such a factor is included into our model.
\end{abstract}

Keywords: Childbearing, Sweden

\section{Introduction}

From previous studies on childbearing in Sweden we know that the relative risk of a mother at parity two to give birth is much lower than it is for a mother at parity one (see, for example, Andersson, 1999). In a society with a strong two-child norm the third birth is a step in the life course where a mother has a clear option to not proceed with further childbearing. Still between 40 and 50 percent of Swedish two-child mothers eventually proceed to give birth to a third child (Martinelle 1992). Andersson (1999) further shows that the relative risk of giving birth to an additional child is not appreciably lower for a three-child mother than it is for the two-child mother: third- and fourth-birth intensities have been almost identical to each other during the last four decades of the twentieth century. The reason for such a similarity is probably that three-child mothers are a rather select group of women who have shown already that they are fairly birth prone. For higher birth orders we may expect to find even higher birth intensities as the group of mothers becomes increasingly select as the parity increases. In a French study, Rallu and Toulemon (1994) showed that this effect at least was evident for the parity progression of four-child mothers in France. 
In the present study, we use Swedish register data to calculate relative risks of giving birth for mothers with different numbers of children. We study the propensity to continue the childbearing beyond child number two and pay special attention to births of those higher orders that are rarely studied in standard demographic analyses. We will examine whether we find any selection effects in the propensity to give birth, and guess that the birth risk of mothers with many children possibly increases with the birth order. Finally, we will include a specific factor in our models that explicitly picks up the effect of unobserved heterogeneity among the women. This will enable us to see to what extent the effect of the number of children on the propensity to give birth is modified when that heterogeneity is controlled for.

\section{Data and method}

The data for our calculations are derived from the Swedish population register system, which covers the whole Swedish population and its vital events with a very high degree of accuracy. From Statistics Sweden we have got access to information about the childbearing histories of all women born in Sweden in 1925 and later ${ }^{1}$. Childbearing histories of immigrant women have not been available for this investigation. In our analysis, we have studied subsequent childbearing after the arrival of a second child. Out of 2.8 million women in the register we accessed, 1.370.000 had attained parity two before the end of 1997, which is our last year of observation. We have followed each of these mothers until the occurrence of a death, emigration, the attainment of age 50, or the end of 1997, whichever comes first. We have censored an observation at a multiple birth and after a ten-year open birth interval. With these restrictions, we have obtained more than 710.000 births at orders three or above, as specified in Table 1.

Table 1. Number of registered births of order 3 and above, for women born in Sweden in 1925 and later

\begin{tabular}{lr}
\hline Number of births & $\mathrm{N}$ \\
$3^{\text {rd }}$ births & 511.302 \\
$4^{\text {th }}$ births & 138.718 \\
$5^{\text {th }}$ births & 39.580 \\
$6^{\text {th }}$ births & 13.069 \\
$7^{\text {th }}$ births & 4.680 \\
$8^{\text {th }}$ births & 1.784 \\
$9^{\text {th }}$ births & 733 \\
$10^{\text {th }}$ births & 355 \\
$11^{\text {th }}$ births & 149 \\
$12^{\text {th }}$ and above & 114 \\
\hline
\end{tabular}

\footnotetext{
${ }^{1}$ This holds for women who were Swedish citizens in 1960 or who were born in Sweden after that year.
} 
Eyeballing the crude numbers of births by birth order we see that these numbers decrease in a rather regular way as the birth order increases. We note that at each birth order the number of registered births is about a third of the recorded number at the immediately preceding birth order. On a closer look, we also note that these fractions tend to increase with increasing birth order.

In this study, we will estimate a measure of the propensity to give birth at the various birth orders, where we can relate the numbers of births properly to exposure times of risk and where we also can control for the effects of a few other basic demographic variables. Time elapsed since previous birth and age of mother are the most important of such variables. We compute our measure by estimating an intensity-regression, or event-history, model where the intensity of giving birth for individual $i$ is described by the following formula:

$\mu_{i}(t)=\mu_{0}(t) \exp \left\{\beta_{1} x_{1 i}+\beta_{2} x_{2 i}\right\}$

Here, $\mu_{0}(t)$ is the baseline intensity in our model, representing the effect of time since previous birth. We let $\mathrm{x}_{1}$ be the birth order, which is our main variable of interest and which at each stage of a woman's childbearing career is treated as a fixed covariate. The variable $\mathrm{x}_{2}$ is the age group of the mother, fixed at each previous birth with the categories "less than 25 years, 25-30 years, 31-36 years, and 37 years or above". Both regressors are represented by a series of binary variables and their coefficients $\beta_{1}$ and $\beta_{2}$ are corresponding parameter vectors. We estimate the parameters $\beta_{j}$ for each level of the variables $x_{j}$ and present the exponentiated values of $\beta_{\mathrm{j}}$. Interpreted as relative risks, they show the propensity to give birth for women of each category, relative to a baseline category on the same factor.

In the next step of our calculations, we add an error term to our model in order to account for woman-specific unobserved heterogeneity in the childbearing propensities of mothers:

$\mu_{i}(t)=\mu_{0}(t) \exp \left\{\beta_{1} x_{1 i}+\beta_{2} x_{2 i}+u_{i}\right\}$

We assume a (log-)normally distributed error term $u_{i}$ with standard deviation $\sigma$ which describes differences in the propensity to give birth according to some unobserved characteristics of the mothers. Since we only have two or three explanatory variables in our model, such differences may be due to variation according to a large number of unknown factors. Many of these factors are of a type that makes it difficult to incorporate them as explicit covariates in models of fertility. Part of the variation in childbearing is, for example, due to variation among women in their fecundity, i.e., in their biological characteristics. Other variation stem from differences in attitudes 
and values in that couples with a desire for larger families are more devoted to family building than others. Differences in fertility may also stem from differences in people's ability and motivation to use contraceptive methods. In some settings, religious beliefs may discourage the use of such methods. Economists, like Heckman et al. (1985), may think about unobserved heterogeneity as representing differences among women in their comparative advantages in childrearing.

The addition of an error term to our model may cause changes in the estimated effects of the observed variables. Some authors argue that it is important to account for unobserved heterogeneity in fertility models in order to get an appropriate picture of the "true" effect of observed variables (see, for example, Heckman et al. 1985, and Ribar 1996). In particular, Kravdal $(2001,2002)$ argues that the joint modeling of parity progressions - like we attempt here - is essential when studying the childbearing dynamics of mothers. Yet there is reluctance among demographers to incorporate such factors in childbearing models partly because of problems with identification of robust models (Heckman and Walker 1987). In our specific model, the heterogeneity factor $u_{i}$ is supposed to be a mother-specific characteristic and thus have the same effect at all higher birth orders. The existence of serial correlation between multiple birth spells of some women will probably enhance the possibility of identifying a parameter $\sigma$ for the distribution of $u_{i}$ (see Keiding 1998).

Finally, we note that some of the effect of this unobserved-heterogeneity factor may be reflected in the length of the preceding birth interval. We assume that women with very short birth intervals may also be more birth prone than others, so the inclusion of such a variable may cause the effect of the other variables to change. Heckman and Walker (1987) tell that such a variable often is used as a proxy for unobserved heterogeneity when investigators are reluctant to incorporate an explicit heterogeneity factor in their models.

Our estimation is performed by means of a computer program called aML, developed by Lee Lillard and Stan Panis at the RAND Corporation. With this software, the baseline intensity $\mu_{0}(t)$ is estimated as a piecewise linear spline. As already mentioned, we start our analysis of the propensity to give birth at the arrival of a second child. Second births will not be considered in our modeling since the demographic determinants for such births are different from corresponding determinants for higher-order births (see Andersson 1999). 


\section{Patterns of higher-order childbearing in Sweden}

In Table 2 we present estimated relative risks of giving birth to another child for women at various levels of our demographic variables. Four different models are presented (Models 1 to 4), with different specifications of variables included. All our presented risks are controlled for the additional effect of time since previous birth. The pattern of this baseline intensity (from our model 2) is shown in Figure 1. It has a similar profile in all our four models.

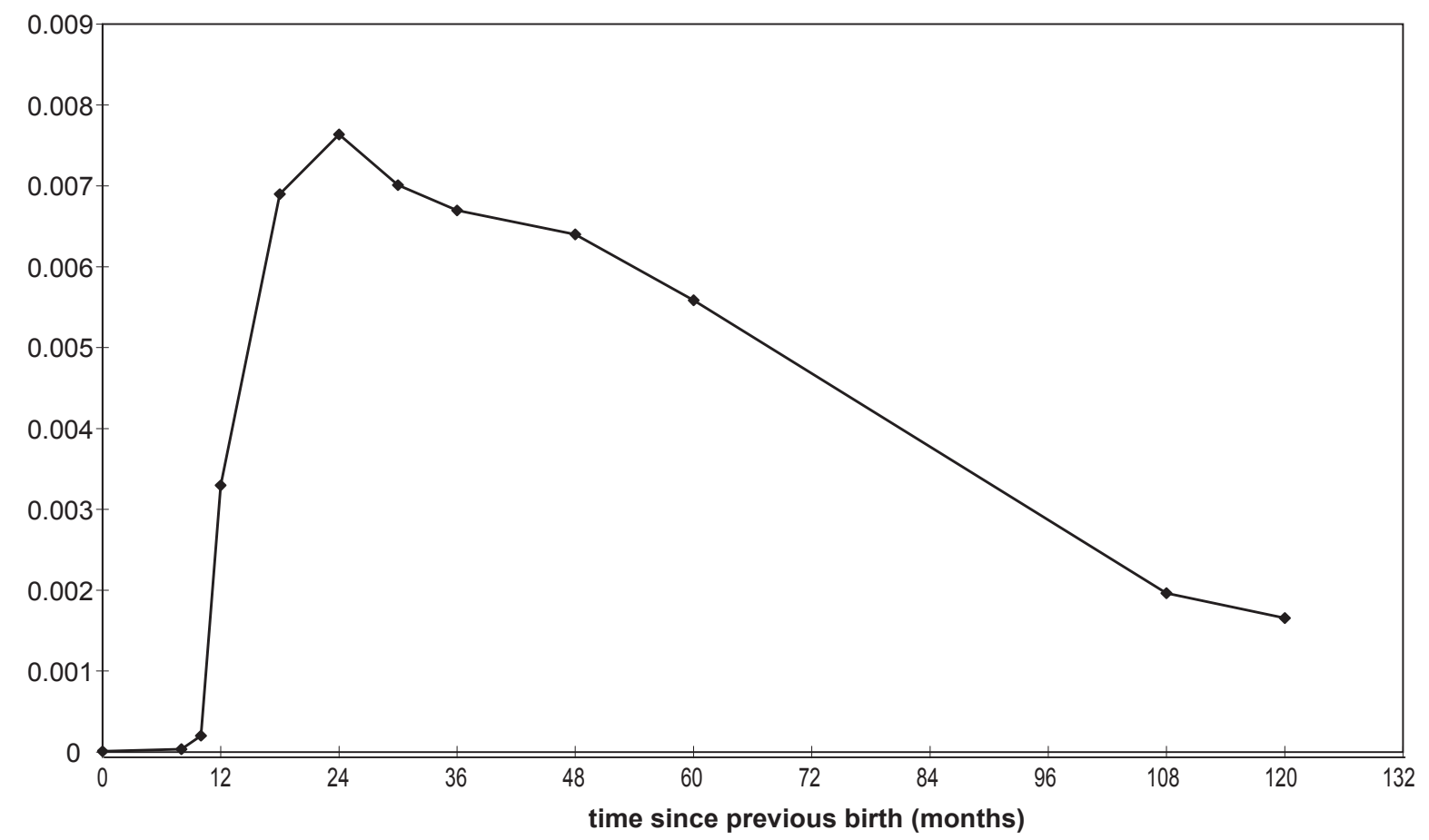

Figure 1. Childbearing intensity, by time since previous birth, Swedish mothers with two or more children

Model 2 is our basic demographic model and it shows the relative risk of giving birth to an additional child, by birth order, standardized for the effect of age of mother and time since previous birth. The model demonstrates that the propensity to give birth increases strongly at higher birth orders, ceteris paribus. While the birth propensity at parity three is around ten percent lower than the propensity at parity two, the risk subsequently increases rapidly with increasingly advanced parity. The relative risk for a fifth birth is 17 percent higher than that for the third birth, the risk for a sixth birth is more than 70 percent higher, that of an eighth birth is three times as high, and the risk of giving birth to a tenth child is almost six times as high as the corresponding risk for the third birth. The relative risk of giving birth is also presented for different ages. As shown by Andersson (1999), there is a strong age gradient in the propensity to give birth to further children after a second birth. The relative risk of childbearing is much lower the older the mother is. Our Model 1 is here presented in order to demonstrate the importance 
of accounting for the effect of that age when estimating birth risks by order of birth. Since births at the very high orders can only occur at rather advanced ages, it is important to account for the fact that the general propensity to give birth then normally is rather low. Otherwise we underestimate the true effect of the propensity to higherorder childbearing, as in Model 1.

Table 2: Relative risk of giving birth to an additional child, by birth order, etc., for mothers in Sweden. Standardized for time since previous birth.

\begin{tabular}{|c|c|c|c|c|}
\hline Birth order & Model 1 & Model 2 & Model 3 & Model 4 \\
\hline 3rd birth & 1 & 1 & 1 & 1 \\
\hline 4th birth & 0.70 & 0.89 & 0.69 & 0.88 \\
\hline 5th birth & 0.75 & 1.17 & 0.71 & 1.09 \\
\hline 6th birth & 0.92 & 1.72 & 0.82 & 1.53 \\
\hline 7th birth & 1.04 & 2.24 & 0.85 & 1.89 \\
\hline 8th birth & 1.18 & 2.94 & 0.90 & 2.38 \\
\hline 9th birth & 1.37 & 3.82 & 0.94 & 2.97 \\
\hline 10th birth & 1.86 & 5.88 & 1.22 & 4.48 \\
\hline 11th birth & 1.44 & 4.95 & 0.80 & 3.59 \\
\hline 12th birth and above & 1.63 & 6.67 & 0.81 & 4.75 \\
\hline \multicolumn{5}{|c|}{ Age of mother (at previous birth) } \\
\hline up to 24 years & & 1.75 & 1.81 & 1.53 \\
\hline $25-30$ years & & 1 & 1 & 1 \\
\hline $31-36$ years & & 0.51 & 0.50 & 0.56 \\
\hline 37 or above & & 0.20 & 0.19 & 0.24 \\
\hline \multicolumn{5}{|c|}{ Length of preceding birth interval } \\
\hline up to 18 months & & & & 1.82 \\
\hline 19-30 months & & & & 1.47 \\
\hline $31-60$ months & & & & 1 \\
\hline 61 months or more & & & & 0.87 \\
\hline Heterogeneity & & & $\sigma=0.63$ & \\
\hline
\end{tabular}

In our Model 3, we proceed with the addition of a factor that explicitly accounts for unobserved heterogeneity among the mothers. The inclusion of such a factor completely erases all the effect of birth order on the propensity to give birth. The relative risk of childbearing for a mother with many children is now slightly below that of a two-child mother and this holds for practically all higher birth orders. See Figure 2 for a visual comparison of the relative risks from Models 2 and 3. We conclude that there is evidence of a strong selection into higher-order childbearing. The specific factor for unobserved heterogeneity introduced in Model 3 manages to catch such selection effects very well. We also note that the estimated effects of the other two variables are not much affected by the inclusion of this unobserved-heterogeneity factor. 


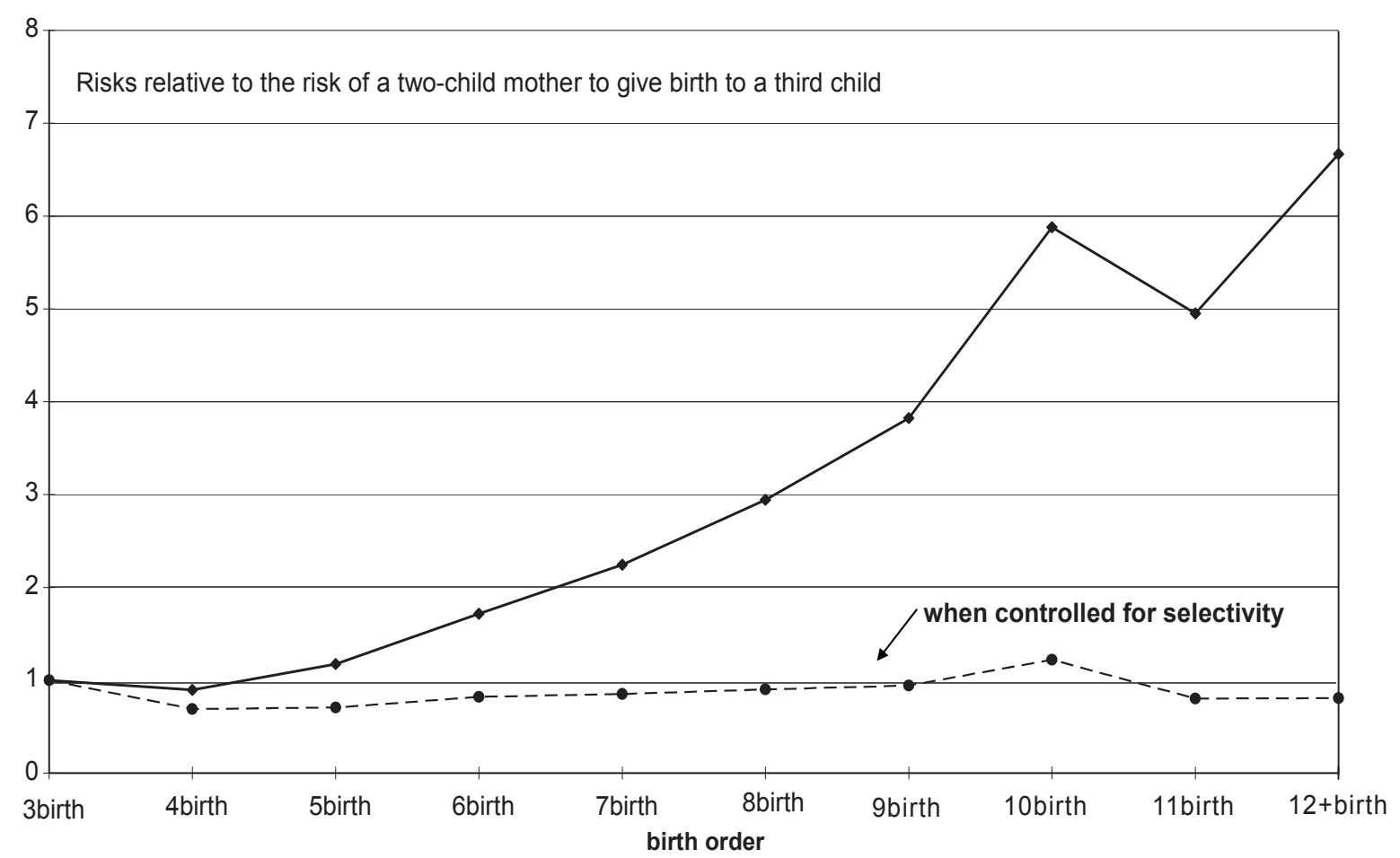

Figure 2. Relative risk of giving birth to an additional child, by birth order, standardized for age of mother and time since previous birth. Swedish mothers with two or more children.

Finally, in Model 4, we substitute the factor which represents unobserved heterogeneity with an observable variable, namely the length of the preceding birth interval. The inclusion of this factor results in a small reduction from Model 2 in the positive gradient in the birth risks by birth order, suggesting that the new variable can catch some but not all of the effects of heterogeneity among mothers with many children. We have also tried to estimate a model including both this birth-interval variable and the factor for unobserved heterogeneity but such a model did not manage to converge in aML. Evidently, these two variables are related to each other in a manner that makes it difficult to disentangle the separate effect of each of the two.

\section{Conclusions}

In this paper, we have shown that there are strong selection effects into higher-order childbearing. We find a strong positive gradient in birth risks by birth order. We have also shown that the inclusion of a factor that explicitly accounts for unobserved heterogeneity totally erases this gradient. This should be encouraging to those who stress the need for controlling for unobserved heterogeneity in fertility models. 


\section{Acknowledgements}

The author is grateful to Statistics Sweden for providing the raw data used in this analysis, and to colleagues in demography for the interest shown in this study. Financial support from the late Swedish Council for Research in the Humanities and the Social Sciences is gratefully acknowledged.

\section{References}

Andersson, G. 1999. Childbearing trends in Sweden 1961-1997. European Journal of Population 15: 1-24.

Heckman, J., and Walker, J. 1987. Using goodness of fit and other criteria to choose among competing duration models: A case study of Hutterite data. In Clogg, C., (ed.) Sociological Methodology 17: 247-307. American Sociological Association.

Heckman, J., Hotz, J., and Walker, J. 1985. New evidence on the timing and spacing of births. The American Economic Review, Papers and Proceedings 75: 179-184.

Keiding, N. 1998. Selection effects and nonproportional hazards in survival models and models for repeated events. Research Report 98/11. Department of Biostatistics, University of Copenhagen.

Kravdal, Ø. 2001. The high fertility of college educated women in Norway: An artefact of the separate modelling of each parity transition. Demographic Research 5: 187216. Available http://www.demographic-research.org/Volumes/Vol5/6.

Kravdal, Ø. 2002. Is the previously reported increase in second- and higher-order birth rates in Norway and Sweden from the mid-1970s real or a result of inadequate estimation methods? Demographic Research 6: 241-262. Available http://www. demographic-research.org/Volumes/Vol6/9.

Martinelle, S. 1992. Fruktsamhet ur ett livsperspektiv. Demografiska rapporter 1992:1. Statistics Sweden, Stockholm.

Rallu, J.-L. and Toulemon, L. 1994. Reflective commentaries: Period fertility measures, the authors' reply. Population: An English Selection 6: 123-130.

Ribar, D. 1996. The effects of teenage fertility on young adult childbearing. Journal of Population Economics 9: 197-218. 